\title{
TREATMENT OF CLOSE APPROACHES \\ IN THE NUMERICAL INTEGRATION OF THE \\ GRAVITATIONAL PROBLEM OF $N$ BODIES
}

D. G. BETTIS AND V.SZEBEHELY

The University of Texas at Austin

\begin{abstract}
One of the main difficulties encountered in the numerical integration of the gravitational $n$-body problem is associated with close approaches. The singularities of the differential equations of motion result in losses of accuracy and in considerable increase in computer time when any of the distances between the participating bodies decreases below a certain value. This value is larger than the distance when tidal effects become important, consequently, numerical problems are encountered before the physical picture is changed. Elimination of these singularities by transformations is known as the process of regularization. This paper discusses such transformations and describes in considerable detail the numerical approaches to more accurate and faster integration. The basic ideas of smoothing and regularization are explained and applications are given.
\end{abstract}

\section{Introduction}

The equations of motion for the gravitational $n$-body problem are

$$
\frac{\mathrm{d}^{2} r_{i}}{\mathrm{~d} t^{2}}=G \sum_{\substack{i=1 \\ 1 \neq j}}^{n} m_{j} \frac{r_{j}-r_{i}}{R_{i j}^{3}},
$$

where $r_{i}$ is the position vector and $m_{i}$ is the mass of the $i$ th body. When

$$
R_{i j}=\left|r_{i}-r_{j}\right|,
$$

becomes small (during a close encounter of the $i$ th and $j$ th bodies) the above differential equation becomes singular. The purpose of this paper is the formulation of an algorithm such that these equations are regular, as $R_{i j} \rightarrow 0$, thus avoiding any loss of significant digits.

First the regularizing transformation for a single pair of bodies is developed and then this result is extended to $n$ pair of bodies.

\section{Basic Principles and a Simple Example of Regularization}

This section is dedicated to the uninitiated to the mysteries of close approaches and to the astronomer who encounters numerical (or analytical) difficulties when integrating the differential equations of dynamical systems.

The following few didactic lines are neither 'practical' nor pleasing to the purist. The simplest possible two-body encounter shall be investigated and an attempt will be made to convert the reader to the ever-increasing number of believers in the method of regularization. The spotlight will be on the essential aspects and neither 
precision-mathematics nor astronomical applications will be allowed to deviate the attention from the simple and straight-forward matters at hand. The practical aspects bring complications which will be handled in the later sections and the mathematical sophistications are well established and available in the literature.

First, an over-simplified model is described. Second, the problem to be solved is outlined. Third, the roads available to handle the problem are outlined. Fourth, one of the possible avenues is selected and the problem is rendered trivial.

In stellar dynamics and in celestial mechanics collision is a physical, rather than a mathematical problem. The centers of two bodies do not occupy the same place at the same time, at least not while still central Newtonian gravitational forces dominate. Higher order gravitational harmonics present in the description of the gravitational field of a planet, for instance, become important when a satellite is in close approach. And in stellar dynamics, for instance, the tidal forces acting between the members of close binaries alter the central Newtonian gravitational forces. The principal question is whether numerical difficulties are encountered as two bodies approach each other before the law of force changes or alters. The answer to this question is in the affirmative both in the field of celestial mechanics and in stellar dynamics. In other words, the tidal effects are still negligible when the close approach destroys the accuracy and efficienty of the numerical integration if conventional formulations are used.

In spite of the fact that collisions do not occur in stellar dynamics in the mathematical sense, the over-simplified model used here is still a collision-model in order to emphasize the computational difficulties and in order to present a simple problem. If the techniques proposed can handle the numerical problems encountered because of a collision, they certainly will be able to treat close approaches. Consequently, the model has two-point masses in a straight line approaching each other. The assumption of point masses instead of finite bodies is, once again, of no importance and allows concentration on the real issues. As the two bodies move, they will approach their (fixed) center of mass. If $x$ is the distance between the two bodies, the equation describing the dynamics of the problem, with properly selected units, $\left[G\left(m_{1}+m_{2}\right)=1\right]$, becomes

$$
\left(\mathrm{d}^{2} x / \mathrm{d} t^{2}\right)=-\left(1 / x^{2}\right) .
$$

As $x$ decreases and approaches zero, the bodies approach each other (and the center of mass of the system). The integral of energy becomes

$$
\frac{1}{2}(\mathrm{~d} x / \mathrm{d} t)^{2}=(1 / x)+C
$$

where $C$ is the constant of energy and it is evaluated from the initial conditions. For simplicity's sake, the motion shall be started with zero velocity when the bodies are apart at a distance $x_{0}$. Consequently, $C=-\left(1 / x_{0}\right)$.

Both of the above equations show singular behavior as $x \rightarrow 0$. The velocity as well as the acceleration approach infinity. Standard numerical integration techniques fail 
close to the singularity since the accurate evaluation of the right side of the equations is not feasible.

The elimination of such singular behavior is called regularization. The regularized equation has no singularity. It is not obvious that the above equations may be regularized, in fact, regularization of a differential equation is not possible in general. When the elimination of singularities is not feasible, often a smoothing of the singular behavior is introduced. Such a smoothing does not regularize but it may ease the numerical problems encountered during integration. The purposes of regularization and smoothing as applied to the differential equations of stellar dynamics are to enhance the process of numerical integration. Consequently, certain regularization techniques which are successful from an analytical point of view may not be satisfactory at all from the point of view of the numerical analyst.

Regularizations and smoothings are accomplished by transformations of the variables occurring in the differential equations of motion. The original regularization of the problem of two bodies as proposed by Sundman (1913) used transformations of the independent (time) variable only. It is recognized today that such transformations while rendering the equations of motion regular, do not offer the best formulation for numerical work. Transformations involving both the dependent and the independent variables are utilized to put the pertinent equations of motion in their optimum form for numerical integration.

Returning to Equations (2) and (3), first a transformation of the time will be introduced to show its effect in the simplest case. The new independent variable is introduced through a differential relation, following Sundman:

$$
\mathrm{d} \tau=\mathrm{d} t / x .
$$

Note that the new 'time' $\tau$ depends on the original time $t$ as well as on the dependent variable $x$. Equation (4) is suggestive of a technique for changing the step-size or time-step during numerical integration. As $x$ becomes smaller, the time-step of integration, $\Delta t$, decreases also in order to accommodate the large changes occuring on the right hand side of the equations to be integrated. In the method of using variable time-steps, no new variable is introduced, but, if one were to appear, it would abide by Equation (4). As $x$ and $\Delta t$ decrease during integration, their ratio may be smooth, or even constant. Consequently, if the variable $\tau$ were used in the differential equations of motion instead of the variable $t$, the steps $(\Delta \tau)$ used may be more even. The essential difference between integration with variable time steps and regularization is that in the latter technique the differential equations are rewritten in terms of the new independent variable. After the regularizing transformations the new equations are integrated. The method of integrating with variable time steps does not change the equations to be integrated; it does not eliminate the singularity; in fact, it does not even smooth the right side of the equations to be integrated. Such a technique must necessarily fail when a singularity is encountered, since as the bodies approach collision, the number of integration steps tends to increase beyond limit. Every step involves truncation and round-off errors, consequently, there is always 
a lower limit on the distance between the bodies below which no integration step can penetrate, still furnishing meaningful results.

Note that Equation (4) itself may introduce a (new) singularity. If the integral of the right side is not a 'proper' one, in other words, if it is not convergent, then the evaluation of the new independent variable $\tau$ may not be accomplished. Leaving the resolution of this problem to a later state, the new 'time' variable will now be introduced into Equations (2) and (3). For this purpose the new 'velocity' $d x / d \tau$ is computed as follows:

$$
x^{\prime}=(\mathrm{d} x / \mathrm{d} \tau)=(\mathrm{d} x / \mathrm{d} t)(\mathrm{d} t / \mathrm{d} \tau)=\dot{x} x
$$

The new velocity is the product of the distance between the bodies $(x)$ and the original velocity. Since, when $x \rightarrow 0, \dot{x} \rightarrow \infty$ as shown by Equation (2), their product $x$ may be a smoothly behaving quantity. Indeed, if Equation (5) is substituted into Equation (3), we have

$$
\left(x^{\prime}\right)^{2}=2 x+2 C x^{2}
$$

as the new, regular equation of energy. As $x \rightarrow 0$, the new velocity approaches zero.

If an exponent of $x$ other than 1 were introduced in Equation (4), the equation for the new velocity would have been different. The selection of the exponent of $x$ in Equation (4) is one of the interesting numerical problems encountered in the numerical integration of the gravitational problem of $n$ bodies and it will be discussed later.

The transformation of Equation (2) is more important than that of Equation (3) since that is the equation of motion to be integrated. In the special and over-simplified example treated in this section, the integral of energy, Equation (3), describes the problem completely and its solution will furnish the solution of Equation (2) also. This special situation is the consequence of treating a motion in one dimension.

The second derivative is evaluated as follows:

or

$$
x^{\prime \prime}=\left(\mathrm{d} x^{\prime} / \mathrm{d} t\right)(\mathrm{d} t / \mathrm{d} \tau)=x(\mathrm{~d} / \mathrm{d} t)(\dot{x} x),
$$

$$
x^{\prime \prime}=\ddot{x} x^{2}+(\dot{x})^{2} x
$$

from which

$$
\ddot{x}=\left[x^{\prime \prime}-\left(x^{\prime}\right)^{2} x^{-1}\right] x^{-2} .
$$

Combining Equations (2) and (7), we obtain

$$
x^{\prime \prime}-\left(\left(x^{\prime}\right)^{2} / x\right)+1=0 \text {. }
$$

Analytically speaking, Equation (8) is regular since

$$
\left(x^{\prime}\right)^{2} / x=2+2 C x
$$

according to Equation (6). Nevertheless, if Equation (6) is not used, because an 
integral of energy is not available, numerical integration of Equation (8) will present difficulties. In the two and three-dimensional cases the elimination of terms corresponding to $\left(x^{\prime}\right)^{2} / x$ in Equation (8) may present serious difficulties. This is one of the main reasons for combining the transformation of the independent variable with a transformation of the dependent variables in more complicated situations.

Substituting now Equation (9) into Equation (8), we obtain the regularized differential equation of motion:

$$
x^{\prime \prime}-2 C x-1=0
$$

First, observe that this equation represents a harmonic oscillator if $C<0$, that is, if the motion is 'elliptic'. By this we mean that if the bodies would be slightly distrubed from their straight line collision orbits, they would describe ellipses and the collision would become a close approach. Note that using the initial conditions postulated before, $C=-1 / x_{0}$. Consequently, $C$ is negative since $x_{0}$ is the initial distance between the bodies.

The solution of Equation (10) with negative energy and satisfying the postulated initial conditions is

$$
x=\left(x_{0} / 2\right)\left(1+\cos \sqrt{\left(2 / x_{0}\right)} \tau\right) .
$$

The tacitly assumed initial condition for the new time variable is that $\tau=0$ when $t=0$.

The dependence of the new velocity on the new time may be obtained either by integrating Equation (6) or by differentiating Equation (11):

$$
x^{\prime}=-\sqrt{\left(x_{0} / 2\right)} \sin \sqrt{\left(2 / x_{0}\right) \tau} .
$$

The relation between the new and original 'time' variables is obtained by integrating Equation (4):

$$
t=\int_{0}^{\tau} x \mathrm{~d} \tau=\left(x_{0} / 2\right)\left[\tau+\sqrt{\left(x_{0} / 2\right)} \sin \sqrt{\left(2 / x_{0}\right) \tau}\right] .
$$

At this point the observant reader will have discovered that $\tau$ is playing the role of the eccentric anomaly and Equation (13) is essentially Kepler's equation. The fact that the eccentric anomaly (and, in fact, also the true anomaly) are regularizing variables is interesting but probably the most significant result is that Equation (10) replaces Equation (2). In other words, the original differential equation of motion containing a $1 / x^{2}$ type singularity is replaced by a linear differential equation without singularity.

In conclusion, we recall that the above example did not intend to be either practical or precise but was arrived at presenting regularization divested from all possible complications and from mathematical niceties. The essential features are the existence 
of a singularity in the original equation of motion, the transformation of the independent variable and the resulting new regular equation of motion.

\section{Jacobian Transformation of the Coordinates}

Assume that bodies $k$ and $l$ are the closest of the $n$ bodies. Defining the vectors $\mathbf{Q}$ and $\mathbf{R}$ by

and

$$
\mathbf{Q}=\frac{m_{k} \mathbf{r}_{k}+m_{l} \mathbf{r}_{l}}{m_{k}+m_{l}}
$$

$$
\mathbf{R}=\mathbf{r}_{k}-\mathbf{r}_{l},
$$

there results two differential equations relating the $k$ th and the $l$ th bodies. This process is discussed in detail by Szebehely (1968) and by Peters (1968) where it is shown that the equations of motion in this (Jacobian) coordinate system become

$$
\ddot{\mathbf{Q}}=\frac{1}{m_{k}+m_{l}} \sum_{\substack{i=1 \\ i \neq k \\ i \neq l}}^{n} m_{i}\left[m_{k} \frac{\mathbf{r}_{i}-\mathbf{r}_{k}}{R_{i k}^{3}}+m_{l} \frac{\mathbf{r}_{i}-\mathbf{r}_{i}}{R_{i l}^{3}}\right]
$$

and

$$
\ddot{\mathbf{R}}=-\left(m_{k}+m_{l}\right)_{R_{k l}^{3}}^{\boldsymbol{r}_{k}-\boldsymbol{r}_{l}}+\sum_{\substack{i=1 \\ i \neq k \\ i \neq l}}^{n} m_{i}\left[\frac{\boldsymbol{r}_{i}-\boldsymbol{r}_{k}}{R_{i k}^{3}}-\frac{\boldsymbol{r}_{i}-\boldsymbol{r}_{l}}{R_{i l}^{3}}\right],
$$

or

$$
\ddot{\mathbf{R}}=-\left(m_{k}+m_{l}\right)\left(\mathbf{R} / R^{3}\right)+\mathbf{F},
$$

where

$$
\mathbf{F}=\sum_{\substack{i=1 \\ i \neq k \\ i \neq l}}^{n} m_{i}\left[\frac{\mathbf{r}_{i}-\mathbf{r}_{k}}{R_{i k}^{3}}-\frac{\mathbf{r}_{i}-r_{l}}{R_{i l}^{3}}\right]
$$

Equation (15) will not suffer from a near singularity since it does not contain $R_{k l}$. Thus it may be considered as an auxiliary equation and solved with the other equations of motion. In order to determine $\mathbf{r}_{k}$ and $\mathbf{r}_{l}$ from Equations (14), $\mathbf{R}$ has to be found. This will be accomplished by a regularizing transformation. If we have $m$ distinct pair of bodies that are near each other, then $m$ transformations according to Equations (14) may be performed to yield a set of equations of the form (15) and (16). This gives us the capability of handling $m$ close approaches.

\section{Smoothing Transformations}

First we will consider transformations of only the independent variable:

$$
\mathrm{d} t=g \mathrm{~d} \tau
$$


where $g$ is some function and $\tau$ is the new independent variable. If

$$
g=R^{\alpha}
$$

where $\alpha$ is an unspecified constant, then

$$
(\mathrm{d} / \mathrm{d} t)=\left(1 / R^{\alpha}\right)(\mathrm{d} / \mathrm{d} \tau)
$$

and

$$
\left(\mathrm{d}^{2} / \mathrm{d} t^{2}\right)=\left(1 / R^{2 \alpha}\right)\left(\mathrm{d}^{2} / \mathrm{d} \tau^{2}\right)-\left(\alpha / R^{1+2 \alpha}\right)(\mathrm{d} R / \mathrm{d} \tau)(\mathrm{d} / \mathrm{d} \tau)
$$

Thus, Equation (17) becomes

$$
\mathbf{R}^{\prime \prime}-\frac{\alpha}{R} \mathbf{R}^{\prime} R^{\prime}+\frac{\left(m_{k}+m_{l}\right)}{R^{3-2 \alpha}} \mathbf{R}=R^{2 \alpha} \mathbf{F},
$$

where the prime denotes differentiation with respect to the new independent variable $\tau$.

Expressed as a first order system, Equation (19) becomes

$$
\begin{aligned}
& \mathbf{R}^{\prime}=\mathbf{S} R^{\alpha} \\
& \mathbf{S}^{\prime}=\left[-\frac{\left(m_{k}+m_{l}\right)}{R^{3}} \mathbf{R}+\mathbf{F}\right] R^{\alpha} .
\end{aligned}
$$

No matter what value of $\alpha$ is selected, there still remains a singularity in Equation (19). However, even though the singularity has not been removed, its severity has been reduced, i.e., the term $R^{3}$ appears in the denominator of Equation (17), while in Equation (19), with $\alpha=\frac{3}{2}$, there is only a factor of $R$ as the divisor. A transformation of this type that only reduces the effect of the singularity will be referred to as a smoothing transformation.

For unperturbed Keplerian motion, the choice $\alpha=1$ corresponds to the use of the eccentric anomaly as the independent variable, and $\alpha=2$ is equivalent to using the true anomaly. For perturbed Keplerian motion numerical experiments indicate that the choice $\alpha=\frac{3}{2}$ is better than $\alpha=1$ or $\alpha=2$. In fact, numerical investigations of orbits of artificial satellites show that the true anomaly has advantages near perigee, and that near apogee the eccentric anomaly is more efficient. Therefore, the use of $\alpha=\frac{3}{2}$ may be considered a compromise for the sallite problem. Experiments with $n$-body problems described by Szebehely and Bettis (1970) corroborate these findings and demonstrate that the selection $\alpha=\frac{3}{2}$ is the most advantageous choice for the smoothing transformation given by Equation (18).

Other choices for the smoothing function $g$ have been suggested by various investigators. Szebehely (1967) discusses the use of the inversion of the velocity vector as the function $g$. Heggie (1970) has recently investigated the use of the potential energy and of the kinetic energy for $g$. The classical choice by Sundman (1912) is $\alpha=1$ which regularizes the problem of two bodies but leaves much to be desired regarding its applicability to numerical work. 


\section{Regularizing Transformations}

\section{A. MOTION IN TWO DIMENSIONS}

In order to remove the singularity in Equation (17) that occurs when $R$ becomes small as well as to offer a method useful for numerical work, Levi-Civita (1903) introduced a coordinate transformation in addition to a transformation of the independent variable for two-dimensional motion. Kustaanheimo and Stiefel (1965) generalized Levi-Civita's transformation to the case when motion takes place in three dimensions.

Levi-Civita proposed Sundman's transformation for the independent variable:

$$
\mathrm{d} t=R \mathrm{~d} \tau .
$$

This gives Equation (19) with $\alpha=1$ as expected:

$$
\mathbf{R}^{\prime \prime}-\frac{\mathbf{R}^{\prime}}{R} \mathbf{R}^{\prime}+\frac{\left(m_{k}+m_{l}\right)}{R} \mathbf{R}=R^{2} \mathbf{F}
$$

In addition, Levi-Civita introduced the coordinate transformation

$$
\begin{aligned}
& R_{1}=u_{1}^{2}-u_{2}^{2}, \\
& R_{2}=2 u_{1} u_{2},
\end{aligned}
$$

where $R_{1}$ and $R_{2}$ are the components of $\mathbf{R}$, and $u_{1}$ and $u_{2}$ are the new dependent variables. The transformation given by Equations (23) may be written as

$$
\mathbf{R}=\mathscr{L}(\mathbf{u}) \mathbf{u},
$$

where

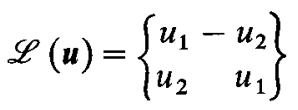

and $\mathbf{u}\left(u_{1}, u_{2}\right)$.

Note that Levi-Civita's transformation may be written as

$$
z=w^{2},
$$

where $z=R_{1}+i R_{2}$ and $w=u_{1}+i u_{2}$ are complex vectors. In the following, with one exception, the matrix formulation is used because of its potential for generalization to higher dimensions. While the complex notation renders an opportunity to be generalized to four-dimensions by quarternions, it was felt that a straight forward, real matrix notation might be more widely understood without special preparation.

As the idea of the transformation of the independent as well as the dependent variables is introduced, a remark regarding the relation between these might be appropriate. Equation (22), the new form of the equation of motion, is the result of transforming only the independent variable. This equation may be considerably simplified or complicated by transforming also the dependent variables. Besides 
regularization, a possible simplification and stabilization of the equations is desirable. In general, the two transformations, using complex notation, may be written as

$$
\mathrm{d} t=g(z) \mathrm{d} \tau
$$

and

$$
z=f(w) .
$$

The proper selection of the function $f(w)$ which introduces the new dependent variable is crucial, since the form of the function $g(z)$ is well established. The choice of $f$, once $g$ is given, is arbitrary in principle. In order to obtain a simple form of the transformed equations of motion, it may be shown (Szebehely, 1966) that the relation

$$
g=a\left|\frac{\mathrm{d} f}{\mathrm{~d} w}\right|^{2}
$$

must be satisfied. Here $a$ is an arbitrary real constant. It should be emphasized that satisfying this relation is not pertinent to the regularization of the equation of motion but it has considerable practical importance.

The afore-mentioned transformation of the time is

$$
g=R=\sqrt{R_{1}^{2}+R_{2}^{2}}=|z|
$$

and Levi-Civita's transformation is $z=w^{2}$. Consequently,

$$
\left|\frac{\mathrm{d} f}{\mathrm{~d} w}\right|=2|w|
$$

and the relation to be satisfied between $f$ and $g$ is

$$
g=4 a|w|^{2},
$$

or

$$
g=4 a|z| .
$$

Comparing this with the function $g=R=\sqrt{R_{1}^{2}+R_{2}^{2}}$ used for the time-transformation as given above or by Equation (21), we have $a=\frac{1}{4}$.

Now, we return to Equation (22) and introduce the new dependent variables given by Equations (23). First, we compute the derivative of $R$. Differentiating Equation (24) with respect to the new independent variable we obtain

$$
\mathbf{R}^{\prime}=2 \mathscr{L}(\mathbf{u}) \mathbf{u}^{\prime} .
$$

From Equations (23) follows the important relation

$$
R=u_{1}^{2}+u_{2}^{2}=\left(R_{1}^{2}+R_{2}^{2}\right)^{1 / 2},
$$

i.e., in the $\mathbf{u}$-space the expression for the relative distance $R$ does not require the 
calculation of a square root. Furthermore, angles at the origin of the R-space are doubled in the new u-space. Consequently, if in the R-space one body makes one revolution about the other, then in the u-space, this body will make only one-half of a revolution. The absence of the computation of the square root, and the halving of the angles at the origin result in considerable computational advantage when the Levi-Civita transformation, or its generalization the Kustaanheimo-Stiefel transformation is used.

Levi-Civita's matrix $\mathscr{L}(\mathbf{u})$ has the following properties (Stiefel and Scheifele, 1970):

$$
\begin{aligned}
& \mathscr{L}^{T}(\mathbf{u}) \mathscr{L}(\mathbf{u})=R I, \\
& \mathscr{L}^{\prime}(\mathbf{u})=\mathscr{L}\left(\mathbf{u}^{\prime}\right), \\
& \mathscr{L}(\mathbf{u}) \mathbf{v}=\mathscr{L}(\mathbf{v}) \mathbf{u},
\end{aligned}
$$

and

$$
(\mathbf{u} \cdot \mathbf{u}) \mathscr{L}(\mathbf{v}) \mathbf{v}-2(\mathbf{u} \cdot \mathbf{v}) \mathscr{L}(\mathbf{u}) \mathbf{v}+(\mathbf{v} \cdot \mathbf{v}) \mathscr{L}(\mathbf{u}) \mathbf{u}=0
$$

where $I$ is the unit matrix, $\mathbf{u}$ and $\mathbf{v}$ are arbitrary vectors and the scalar product is defined by the notation $(\mathbf{u} \cdot \mathbf{u})$. We proceed now to express Equation (22) in terms of the new dependent variable $\mathbf{u}$. From Equation (25) it follows that

$$
\mathbf{R}^{\prime \prime}=2 \mathscr{L}(\mathbf{u}) \mathbf{u}^{\prime \prime}+2 \mathscr{L}^{\prime}(\mathbf{u}) \mathbf{u}^{\prime},
$$

or

$$
\mathbf{R}^{\prime \prime}=2 \mathscr{L}(\mathbf{u}) \mathbf{u}^{\prime \prime}+2 \mathscr{L}\left(\mathbf{u}^{\prime}\right) \mathbf{u}^{\prime}, \quad \text { since } \mathscr{L}^{\prime}(\mathbf{u})=\mathscr{L}\left(\mathbf{u}^{\prime}\right) .
$$

Using this expression for $\mathbf{R}^{\prime \prime}$ and Equations (24) and (25), Equation (22) becomes

$$
\begin{aligned}
2(\mathbf{u} \cdot \mathbf{u}) \mathscr{L}(\mathbf{u}) \mathbf{u}^{\prime \prime}+2(\mathbf{u} \cdot \mathbf{u}) \mathscr{L}\left(\mathbf{u}^{\prime}\right) \mathbf{u}^{\prime}-4\left(\mathbf{u} \cdot \mathbf{u}^{\prime}\right) \mathscr{L}(\mathbf{u}) \mathbf{u}^{\prime}+ \\
+\left(m_{k}+m_{l}\right) \mathscr{L}(\mathbf{u}) \mathbf{u}=(\mathbf{u} \cdot \mathbf{u})^{3} \mathbf{F},
\end{aligned}
$$

where $R^{\prime}$ was eliminated by the relation

$$
R^{\prime}=2\left(\mathbf{u} \cdot \mathbf{u}^{\prime}\right)
$$

With the aid of the Equation (26d), Equation (27) may be expressed as

$$
2(\mathbf{u} \cdot \mathbf{u}) \mathscr{L}(\mathbf{u}) \mathbf{u}^{\prime \prime}-2\left(\mathbf{u}^{\prime} \cdot \mathbf{u}^{\prime}\right) \mathscr{L}(\mathbf{u}) \mathbf{u}+\left(m_{k}+m_{l}\right) \mathscr{L}(\mathbf{u}) \mathbf{u}=(\mathbf{u} \cdot \mathbf{u})^{3} \mathbf{F} .
$$

Multiplying this expression by $\mathscr{L}^{-1}(\mathbf{u})$ and by using Equation (26a), we obtain

$$
\mathbf{u}^{\prime \prime}+\frac{\left(m_{k}+m_{l}\right)-2\left(\mathbf{u}^{\prime} \cdot \mathbf{u}^{\prime}\right)}{2(\mathbf{u} \cdot \mathbf{u})} \mathbf{u}=\frac{(\mathbf{u} \cdot \mathbf{u})}{2} \mathscr{L}^{T}(\mathbf{u}) \mathbf{F} .
$$

Observe that the coefficient of $\mathbf{u}$ is one-half of the negative of the two-body binding energy per unit mass $h$, since, in the $\mathbf{R}$-space,

$$
h=\frac{(\dot{\mathbf{R}} \cdot \dot{\mathbf{R}})}{2}-\frac{\left(m_{k}+m_{l}\right)}{R}<0
$$


and in the $\mathbf{v}$ space

$$
h=\frac{2\left(\mathbf{u}^{\prime} \cdot \mathbf{u}^{\prime}\right)-\left(m_{k}+m_{l}\right)}{(\mathbf{u} \cdot \mathbf{u})} .
$$

The transformed equation for $\mathbf{R}$ may now be written as

$$
\mathbf{u}^{\prime \prime}-\frac{h}{2} \mathbf{u}=\frac{(\mathbf{u} \cdot \mathbf{u})}{2} \mathscr{L}^{T}(\mathbf{u}) \mathbf{F} .
$$

Equation (29b) contains a singularity. Consequently, if it is used to compute the binding energy, $h$, then Equation (30) will have a singularity. A regular differential equation may be obtained for the binding energy. If Equation (17) is multiplied by $\dot{\mathbf{R}}$ we have

$$
(\dot{\mathbf{R}} \cdot \ddot{\mathbf{R}})=-\frac{\left(m_{k}+m_{l}\right)}{R^{3^{3}}}(\dot{\mathbf{R}} \cdot \mathbf{R})+(\dot{\mathbf{R}} \cdot \mathbf{F})
$$

Since

$$
(\dot{\mathbf{R}} \cdot \mathbf{R})=\dot{R} R
$$

Equation (31) becomes

$$
\dot{\mathbf{R}} \cdot \ddot{\mathbf{R}}+\left(m_{k}+m_{l}\right)\left(\dot{R} / R^{2}\right)=\dot{\mathbf{R}} \cdot \mathbf{F},
$$

or

$$
\frac{\mathrm{d}}{\mathrm{d} t}\left[\frac{\dot{\mathbf{R}} \cdot \dot{\mathbf{R}}}{2}-\frac{\left(m_{k}+m_{l}\right)}{R}\right]=\dot{\mathbf{R}} \cdot \mathbf{F}
$$

The expression in the brackets above is the two-body binding energy per unit mass, therefore

$$
(\mathrm{d} h / \mathrm{d} t)=\dot{\mathbf{R}} \cdot \mathbf{F},
$$

or,

$$
h^{\prime}=\mathbf{R}^{\prime} \cdot \mathbf{F} \text {. }
$$

In the u-space this becomes

$$
h^{\prime}=2\left(\mathscr{L}(\mathbf{u}) \mathbf{u}^{\prime} \cdot \mathbf{F}\right)
$$

or,

$$
h^{\prime}=2\left(\mathbf{u}^{\prime} \cdot \mathscr{L}^{T}(\mathbf{u}) \mathbf{F}\right)
$$

Equations (21), (30), and (32) form a system of regular differential equations which may be solved for $\mathbf{u}$ and for the time, $t$. Then $\mathbf{R}$ may be obtained by using Equation (24).

\section{B. MOTION IN THREE DIMENSIONS}

In order to extend Levi-Civita's transformation to three dimensions, Kustaanheimo 
and Stiefel (1965) introduced two four-dimensional vectors $\mathbf{R}$ and $\mathbf{u}$ which are connected by

$$
\mathbf{R}=\mathscr{L}(\mathbf{u}) \mathbf{u},
$$

where

$$
\mathscr{L}(\mathbf{u})=\left(\begin{array}{rrrr}
u_{1} & -u_{2} & -u_{3} & u_{4} \\
u_{2} & u_{1} & -u_{4} & -u_{3} \\
u_{3} & u_{4} & u_{1} & u_{2} \\
u_{4} & -u_{3} & u_{2} & -u_{1}
\end{array}\right)
$$

and where

$$
\mathbf{R}=\left(\begin{array}{l}
R_{1} \\
R_{2} \\
R_{3} \\
R_{4}
\end{array}\right), \quad u=\left(\begin{array}{l}
u_{1} \\
u_{2} \\
u_{3} \\
u_{4}
\end{array}\right)
$$

The components of $R$ are

$$
\left.\begin{array}{l}
R_{1}=u_{1}^{2}-u_{2}^{2}-u_{3}^{2}+u_{4}^{2}, \\
R_{2}=2\left(u_{1} u_{2}-u_{3} u_{4}\right) \\
R_{3}=2\left(u_{1} u_{3}+u_{2} u_{4}\right) \\
R_{4}=0
\end{array}\right\}
$$

and its magnitude is

$$
R=\sqrt{\mathbf{R} \cdot \mathbf{R}}=u_{1}^{2}+u_{2}^{2}+u_{3}^{2}+u_{4}^{2} .
$$

Hurwitz (1933) has shown that the generalization of Levi-Civita's transformation to three dimensions is not possible, but that the transformation may be extended to four dimensions. Indeed, by using the four-by-four matrix $\mathscr{L}(\mathbf{u})$, Equations (30) and $(32)$ remain valid.

Since one of the four components of the vector $\mathbf{u}$ is arbitrary, the question arises as to how to select the initial values of the components of $\mathbf{u}$ where $\mathbf{R}$ is given. Adding the first of Equations (33) to Equation (34) we have

$$
u_{1}^{2}+u_{4}^{2}=\frac{1}{2}\left(R_{1}+R\right) \text {. }
$$

Keeping in mind the arbitrariness of one of the components of $u$, we will select $u_{1}$ and $u_{4}$ such that this relation is satisfied. For simplicity we will select either $u_{1}$ or $u_{4}$ identically equal to zero or $u_{1}$ equal to $u_{4}$. From the second and third equations of Equations (33) there results

$$
u_{2}=\frac{R_{2} u_{1}+R_{3} u_{4}}{R_{1}+R}, \quad u_{3}=\frac{R_{3} u_{1}-R_{2} u_{4}}{R_{1}+R} .
$$

When $R_{1} \geqslant 0$, Equations (35a) and (35b) are used to determine the initial values of the components of $u$. In order to avoid the loss of significant digits when $R_{1}<0$, 
it is advantageous to use the set of relations

$$
\begin{aligned}
& u_{2}^{2}+u_{3}^{2}=\frac{1}{2}\left(R-R_{1}\right), \\
& u_{1}=\frac{R_{2} u_{2}+R_{3} u_{3}}{R-R_{1}}, \quad u_{4}=\frac{R_{3} u_{2}-R_{2} u_{3}}{R-R_{1}},
\end{aligned}
$$

choosing either $u_{2}$ or $u_{3}$ arbitrarily.

Once the components of $u$ are determined, then $u^{\prime}$ is determined from Equations (25) and (26a):

$$
\mathbf{u}^{\prime}=(1 / 2 R) \mathscr{L}^{T}(\mathbf{u}) \mathbf{R}^{\prime},
$$

or

$$
\mathbf{u}^{\prime}=\frac{1}{2} \mathscr{L}^{T}(\mathbf{u}) \dot{\mathbf{R}}
$$

To obtain the time, $t$, it is necessary to solve the differential equation

$$
t^{\prime}=R
$$

where $R$ is computed from Equation (34). It is assumed at this point that the differential equation of motion, Equation (30) has been solved, so $\mathbf{u}(\tau)$ is known. An alternative, which may be used either as a check on the accuracy of the computation of $R$ or to obtain $R$ for using it in Equation (38), is to solve a differential equation for $R$. By differentiating Equation (34) twice with respect to $\tau$, there results

$$
R^{\prime \prime}=2\left(\mathbf{u}^{\prime \prime} \cdot \mathbf{u}\right)+2\left(\mathbf{u}^{\prime} \cdot \mathbf{u}^{\prime}\right)
$$

By using Equations (29b) and (30) this becomes

$$
R^{\prime \prime}-2 h R=\left(m_{k}+m_{l}\right)+R\left[\mathbf{u} \cdot \mathscr{L}^{T}(\mathbf{u}) \mathbf{F}\right] .
$$

If the perturbation forces $\mathbf{F}$ are zero, Equations (30) and (39) become the differential equations of a harmonic oscillator which are stable in the Liapunov sense. It is well known that the original equation of motion, Equation (17), for $\mathbf{R}$ is unstable in the Liapunov sense. Since $\mathbf{F}$ is not zero in our $n$-body problem, strict Liapunov stability no longer exists, but the transformed equations represent a much more stable system of differential equations than the original.

\section{Summary of the K-S Formulation}

This section lists the computational steps to be performed when the $\mathrm{K}-\mathrm{S}$ method of three dimensional regularization is used. It is assumed that the components of $\mathbf{R}$ and $\dot{\mathbf{R}}$ are given.

First compute $R$ from

$$
R=\sqrt{R_{1}^{2}+R_{2}^{2}+R_{3}^{2}}
$$


If $R_{1} \geqslant 0$, let $u_{4}=0$, and compute the remaining components of $u$ as:

$$
\begin{aligned}
& u_{1}=\sqrt{\frac{1}{2}\left(R_{1}+R\right)}, \\
& u_{2}=\left(R_{2} / 2 u_{1}\right), \quad u_{3}=\left(R_{3} / 2 u_{1}\right) .
\end{aligned}
$$

If $R_{1}<0$, let $u_{3}=0$ and use the relations

$$
\begin{aligned}
& u_{2}=\sqrt{\frac{1}{2}\left(R-R_{1}\right)} \\
& u_{1}=\left(R_{2} / 2 u_{2}\right), \quad u_{4}=\left(R_{3} / 2 u_{2}\right) .
\end{aligned}
$$

Then to compute $u^{\prime}$ from Equation (37) use

$$
\begin{aligned}
& u_{1}^{\prime}=\frac{1}{2}\left(u_{1} \dot{R}_{1}+u_{2} \dot{R}_{2}+u_{3} \dot{R}_{3}\right), \\
& u_{2}^{\prime}=\frac{1}{2}\left(-u_{2} \dot{R}_{1}+u_{1} \dot{R}_{2}+u_{4} \dot{R}_{3}\right), \\
& u_{3}^{\prime}=\frac{1}{2}\left(-u_{3} \dot{R}_{1}-u_{4} \dot{R}_{2}+u_{1} \dot{R}_{3}\right), \\
& u_{4}^{\prime}=\frac{1}{2}\left(u_{4} \dot{R}_{1}-u_{3} \dot{R}_{2}+u_{2} \dot{R}_{3}\right) .
\end{aligned}
$$

The initial value of the binding energy as given by Equation (29a) is

$$
h=\frac{\dot{\mathbf{R}} \cdot \dot{\mathbf{R}}}{2}-\frac{\left(m_{k}+m_{l}\right)}{R} .
$$

Using the initial values obtained above for $\mathbf{u}, \mathbf{u}^{\prime}$ and $h$, solve the system of differential equations:

$$
\begin{aligned}
u_{1}^{\prime \prime}-\frac{h}{2} u_{1} & =\frac{R}{2}\left(\mathscr{L}^{T} \mathbf{F}\right)_{1}, \\
u_{2}^{\prime \prime}-\frac{h}{2} u_{2} & =\frac{R}{2}\left(\mathscr{L}^{T} \mathbf{F}\right)_{2}, \\
u_{3}^{\prime \prime}-\frac{h}{2} u_{3} & =\frac{R}{2}\left(\mathscr{L}^{T} \mathbf{F}\right)_{3}, \\
u_{4}^{\prime \prime}-\frac{h}{2} u_{4} & =\frac{R}{2}\left(\mathscr{L}^{T} \mathbf{F}\right)_{4}, \\
R^{\prime \prime}-2 h R & =\left(m_{k}+m_{l}\right)+R \sum_{i=1}^{4}\left(\mathscr{L}^{T} \mathbf{F}\right)_{i} U_{i}, \\
h^{\prime} & =2 \sum_{i=1}^{4}\left(\mathscr{L}^{T} F\right)_{i} u_{i}^{\prime} \\
t^{\prime} & =R .
\end{aligned}
$$

Here

$$
\begin{aligned}
& \left(\mathscr{L}^{T} \mathbf{F}\right)_{1}=u_{1} F_{1}+u_{2} F_{2}+u_{3} F_{3}, \\
& \left(\mathscr{L}^{T} \mathbf{F}\right)_{2}=-u_{2} F_{1}+u_{1} F_{2}+u_{4} F_{3}, \\
& \left(\mathscr{L}^{T} \mathbf{F}\right)_{3}=-u_{3} F_{1}-u_{4} F_{2}+u_{1} F_{3}, \\
& \left(\mathscr{L}^{T} \mathbf{F}\right)_{4}=u_{4} F_{1}-u_{3} F_{2}+u_{2} F_{3} .
\end{aligned}
$$


The formulae necessary for the transformation from the $\mathbf{U}$-space to the $\mathbf{R}$-space are

$$
\begin{aligned}
& R_{1}=u_{1}^{2}-u_{2}^{2}-u_{3}^{2}+u_{4}^{2}, \\
& R_{2}=2\left(u_{1} u_{2}-u_{3} u_{4}\right), \\
& R_{3}=2\left(u_{1} u_{3}+u_{2} u_{4}\right),
\end{aligned}
$$

and

$$
\begin{aligned}
& \dot{R}_{1}=\frac{2}{R}\left(u_{1} u_{1}^{\prime}-u_{2} u_{2}^{\prime}-u_{3} u_{3}^{\prime}+u_{4} u_{4}^{\prime}\right), \\
& \dot{R}_{2}=\frac{2}{R}\left(u_{2} u_{1}^{\prime}+u_{1} u_{2}^{\prime}-u_{4} u_{3}^{\prime}-u_{3} u_{4}^{\prime}\right), \\
& \dot{R}_{3}=\frac{2}{R}\left(u_{3} u_{1}^{\prime}+u_{4} u_{2}^{\prime}+u_{1} u_{3}^{\prime}+u_{2} u_{4}^{\prime}\right) .
\end{aligned}
$$

During the solution of the system of differential equations, the following checks may be employed:

(i) the distance

$$
R=\sum_{i=1}^{4} u_{i}^{2}
$$

(ii) the binding energy

$$
h=\frac{2 \sum_{i=1}^{4} u_{i}^{\prime 2}-\left(m_{k}+m_{l}\right)}{R} .
$$

\section{The Use of Several Independent Variables}

Whenever transformations of the independent variable are performed there will be at least two 'time' variables which have to be related. For example, assume that bodies 1 and 2 on one hand and bodies 3 and 4 on the other hand, form two pairs so that these two pairs are not close to each other. Also assume that the other bodies $4,5, \ldots, n$ are far from these pairs. Denoting the relative distance of bodies 1 and 2 by $R_{12}$ and that of bodies 3 and 4 by $R_{34}$, we have for the new independent variables

$$
\mathrm{d} t=R_{12} \mathrm{~d} \tau_{12}=R_{34} \mathrm{~d} \tau_{34} .
$$

By selecting the independent variable with the smallest factor, for example $\tau_{12}$ we may relate the other times to this $\tau_{12}$ by

$$
\mathrm{d} t=R_{12} \mathrm{~d} \tau_{12} \quad \text { and } \mathrm{d} \tau_{45}=\left(R_{12} / R_{34}\right) \mathrm{d} \tau_{12} .
$$

This idea may be extended to any distant pair of bodies. By writing the equations of motion as a system of first order differential equations, the correlation of the different times may be accomplished by multiplying all of the equations by the 
suitable factor of $R_{i j}$. That is, if the original equations of motion,

$$
\left(\mathrm{d}^{2} \mathbf{r}_{i} / \mathrm{d} t^{2}\right)=f_{i}\left(\mathbf{r}_{1}, \ldots, \mathbf{r}_{n}\right)
$$

are written as

$$
\begin{aligned}
\left(\mathrm{d} \mathbf{r}_{1} / \mathrm{d} \tau_{12}\right)=\mathbf{V}_{1}, & \left(\mathrm{~d} \mathbf{V}_{1} / \mathrm{d} \tau_{12}\right)=\mathbf{f}_{1}, \\
\left(\mathrm{~d} \mathbf{r}_{2} / \mathrm{d} \tau_{12}\right)=\mathbf{V}_{2}, & \left(\mathrm{~d} \mathbf{V}_{2} / \mathrm{d} \tau_{12}\right)=\mathbf{f}_{2}, \\
\left(\mathrm{~d} \mathbf{r}_{3} / \mathrm{d} \tau_{34}\right)=\mathbf{V}_{3}, & \left(\mathrm{~d} \mathbf{V}_{3} / \mathrm{d} \tau_{34}\right)=\mathbf{f}_{3}, \\
\left(\mathrm{~d} \mathbf{r}_{4} / \mathrm{d} \tau_{34}\right)=\mathbf{V}_{4}, & \left(\mathrm{~d} \mathbf{V}_{4} / \mathrm{d} \tau_{34}\right)=\mathbf{f}_{4}, \\
\left(\mathrm{~d} \mathbf{r}_{j} / \mathrm{d} t\right)=\mathbf{V}_{j}, & \left(\mathrm{~d} \mathbf{V}_{j} / \mathrm{d} t\right)=\mathbf{f}_{j}, \quad j=5,6, \ldots, n,
\end{aligned}
$$

then by multiplying all but the first four of these equations by the appropriate factors of $R_{i j}$, we obtain the system

$$
\begin{aligned}
& \left(\mathrm{d} \mathbf{r}_{1} / \mathrm{d} \tau_{12}\right)=\mathbf{V}_{1}, \quad\left(\mathrm{~d} \mathbf{V}_{1} / \mathrm{d} \tau_{12}\right)=\mathbf{f}_{1}, \\
& \left(\mathrm{~d} \mathbf{r}_{2} / \mathrm{d} \tau_{12}\right)=\mathbf{V}_{2}, \quad\left(\mathrm{~d} \mathbf{V}_{2} / \mathrm{d} \tau_{12}\right)=\mathbf{f}_{2}, \\
& \left(\mathrm{~d} \mathbf{r}_{3} / \mathrm{d} \tau_{12}\right)=\mathbf{V}_{2}\left(R_{12} / R_{34}\right), \quad\left(\mathrm{d} \mathbf{V}_{3} / \mathrm{d} \tau_{12}\right)=\mathbf{f}_{3}\left(R_{12} / R_{34}\right) \\
& \left(\mathrm{d} \mathbf{r}_{4} / \mathrm{d} \tau_{12}\right)=\mathbf{V}_{4}\left(R_{12} / R_{34}\right), \quad\left(\mathrm{d} \mathbf{V}_{4} / \mathrm{d} \tau_{12}\right)=\mathbf{f}_{4}\left(R_{12} / R_{34}\right) \\
& \left(\mathrm{d} \mathbf{r}_{j} / \mathrm{d} \tau_{12}\right)=\mathbf{V}_{j} R_{12}, \quad\left(\mathrm{~d} \mathbf{V}_{j} / \mathrm{d} \tau_{12}\right)=\mathbf{f}_{j} R_{12} .
\end{aligned}
$$

Thus the single independent variable $\tau_{12}$ may be used during the numerical integration, even though several different time variables were introduced.

\section{Numerical Solution of the Differential Equations}

Traditionally in celestial mechanics, finite difference methods have been used for the numerical solution of differential equations, such as the Störmer-Cowell or the Adams-Bashforth-Moulton methods. More recently methods based on power series gained popularity, such as straight forward Taylor series or recurrent power series. The choise of the particular method is dependent upon the speed and the memory capacity of the electronic computer being used. In general, the limitations of the computer become more critical as the number of bodies increases.

A special problem emerges when numerical integration is performed with $m$ pair of bodies regularized by the $\mathrm{K}-\mathrm{S}$ transformation. Everytime the configuration (i.e., the participating bodies) changes, the $\mathrm{K}-\mathrm{S}$ transformation must be re-initialized. This is of no consequence to the self-starting power series methods, but it is difficult for the multi-step methods which require knowledge of the solution of the differential equations for several previous values of the independent variable. However, if the configuration is rather stable, the $\mathrm{K}-\mathrm{S}$ transformation does not need to be re-initialized, and a starting routine does not need to be called.

In order to reach a compromise between computational accuracy and computer time during the close encounter of $m$ pair of bodies, some criterion must be established as to when to regularize. In general, a close pair should be regularized by the $\mathrm{K}-\mathrm{S}$ transformation only when there is reason to suspect that there is, or will be, a loss of significant digits in the calculation. While establishing such a criterion one must 
remember the most apparent advantages of the $\mathrm{K}-\mathrm{S}$ transformation, (i) regular differential equations for a pair of bodies, (ii) no computation of a square root in order to determine, $R$ and (iii) angles at the origin are doubled. These advantages should be weighed against the following disadvantages: (i) the differential equations are not regular if there are more than two bodies in the encounter, (ii) there are additional differential equations to be solved, and (iii) time consuming transformations to the Jacobian coordinates and to the $\mathrm{K}-\mathrm{S}$ coordinates must be executed and vice-versa.

\section{Numerical Methods}

For an explanation of the use of the finite difference methods we refer the reader to any or all of the following articles: Henrici (1962), Schubart and Stumpff (1966), Aarseth (1970), Bettis (1970), and Stiefel and Bettis (1969).

It has been found that if the number of bodies is of the order of one-hundred to two-hundred, high order Runge-Kutta methods are very efficient when the configuration of the $m$ pair of regularized bodies is rapidly changing. In particular, a RungeKutta method by Fehlberg (1968) has proved very efficient with a CDC 6600 Computer for accurate work as shown by Szebehely and Bettis (1970).

For the sake of the convenience of the reader, Fehlberg's algorithm is outlined. For a system of differential equations of the first order,

$$
(\mathrm{d} y / \mathrm{d} t)=f(t, y)
$$

the solution for the value $t=t_{0}+s$, Fehlberg gives

$$
\begin{aligned}
f_{0} & =f\left(t_{0}, y_{0}\right), \\
f_{\kappa} & =f\left(t_{0}+\alpha_{\kappa} s, \quad y_{0}+s \sum_{\lambda=0}^{\kappa-1} \beta_{\kappa \lambda} f_{\lambda}\right), \quad \kappa=1,2,3, \ldots, 12, \\
y & =y_{0}+s \sum_{k=0}^{12} C_{\kappa} f_{\kappa}+0\left(s^{9}\right),
\end{aligned}
$$

where $s$ is the step-size.

Even though this method requires thirteen evaluations of the function $f(t, y)$ for each step, the precise estimate of the step sizes of Fehlberg's method compensates for this disadvantage. The truncation error for this method is

$$
\frac{41}{840}\left(f_{0}+f_{10}+f_{11}+f_{12}\right) s .
$$

From this relation, the step-size $s$ may be estimated. The reader is referred to the original article by Fehlberg (1968) for further details and for the coefficients $\alpha_{x}, \beta_{x \lambda}$, and $C_{x}$.

\section{Acknowledgments}

Partial support of a Contract No. N00014-67-A-0126-0007 from the Office of Naval Research is acknowledged. Fruitful discussions are acknowledged with great pleasure conducted between Dr S. J. Aarseth, Mr D. Heggie, and the authors. 


\section{References}

Aarseth, S.: 1970, this issue, p. 20.

Bettis, D. G.: 1970, Numer. Math. 14, 421-434.

Fehlberg, E.: 1968, NASA TR R-287, also 1969, Computing 4, 93.

Heggie, D.: 1970, this issue, p. 35.

Henrici, P.: 1962, Discrete Variable Methods in Ordinary Differential Equations, John Wiley and Sons.

Hurwitz, A.: 1933, Math. Werke, Vol. 2, Birkhauser, Basel.

Kustaanheimo, P. and Stiefel, E.: 1965, J. Math. 218, 204.

Levi-Civita, T.: 1903, Ann. Math. 9, 1.

Peters, C. F.: 1968, Bull. Astron. 3, 167.

Schubart, J. and Stumpff, P.: 1966, Veröff. Astron. Rechen-Inst. Heidelberg, No. 18, Karlsruhe.

Stiefel, E. and Bettis, D. G.: 1969, Numer. Math. 13, 154-75.

Stiefel, E. and Scheifele, G.: 1970, Linear and Regular Celestial Mechanics, Springer-Verlag.

Sundman, K. F.: 1912, Acta Math. 35, 105.

Szebehely, V.: 1967, Theory of Orbits, Academic Press, New York.

Szebehely, V.: 1968, Bull. Astron. 3, 91.

Szebehely, V. and Bettis, D. G.: 1970, Astrophys. Space Sci. 13, 365. 